\title{
Central Effect of Some Vasoactive Substances as Observed by the Cross-Circulation in the Dog
}

\author{
Hiroshi Kumagar, M.D., Ph.D.
}

\begin{abstract}
THE topic to be discussed here is the result of a series of experiments concerned with the CNS conducted in the Department of Pharmacology, University of Tokyo. This series of experiments was originally designed to obtain informations on the role of chemical transmitters in the CNS. Though the essential role of adrenergic as well as cholinergic transmitters in the peripheral system is generally accepted, the role of these transmitter substances in the CNS is still obscure and requires further investigations to be elucidated.

We had devised a newer method of cross-circulation of the head using dogs. The original idea and method were reported by J.F. and C. Heymans and coworkers in 1920.
\end{abstract}

\section{MFthods}

In this new type of cross-circulation preparation, the interruption of circulatory pathways inside the spinal column is particularly elaborated. The surgical procedures are carried out by the ventral approach instead of the dorsal one of the precedent workers.

1. Donor Dog. The donor dog was anesthetized with barbiturates or preferably stunned by electroshock applied to the forehead through padded, saline-soaked electrodes; small amounts of muscle relaxant might be concomitantly applied without altering the results due to the subsequent administration of drugs tested.

2. Recipient Dog. The recipient dog weighing about $10 \mathrm{Kg}$. was anesthetized with one of short-acting barbiturates. The circulatory system of the dog was transversely divided at the occipito-atloid level leaving the principal nervous system intact. This operation was done from the ventral approach including ligation of arteries and veins, transection of muscles, and clamping of the longitudinal vertebral sinuses with specially devised hemostats.

The anesthetized recipient dog was fixed on its back, and a T-cannula was inserted into the lower portion of the trachea. In some cases the bilateral vertebral arteries were tied off at their entrance into the transverse foramen of the 6th vertebra. The trachea and esophagus were cut and retroflexed separated from the surrounding tissues. The ventral occipito-atloid ligament was exposed by severing

From Department of Pharmacology, Faculty of Medicine, University of Tokyo, Bunkyoku, Tokyo. This paper was read before the Tokyo Society of Medical Science on Oct. 21, 1960. 
the capitis muscles. At the level of the occipito-atloid joint the following vessels were bilaterally ligated (Fig. 1) : anastomosis between occipital and vertebral arteries, anastomosis between vertebral and cerebrospinal arteries, the vertebral veins, anastomosis between vertebral vein and vertebral sinus, and anastomosis between vertebral sinus and occipital vein. The capsule and ligament between the occipital bone and atlas were removed. The bilateral external and internal jugular veins were also ligated at the occipito-atloid level, and the remaining soft tissues around the neck were transected to the bones with an electric knife except the bilateral carotid arteries and vagosymapthetic nerve trunks. The bilateral vertebral arteries and veins were again ligated at the opening of transverse foramen of the atlas. Thus the circulatory communications between the head and trunk had been cut off leaving the bilateral common carotid arteries and the vessels running inside the spinal column.

The ventral arch of the atlas was removed, and the dens of the epistropheus is eradicated. The ventral portion of the dura mater and arachnoid was removed. After heparinizing the animal a hole was carefully bored through the parietal bone to the transverse sinus on each side of the protuberance just above the superior linea nuchae. Metal cannulae were screwed into both holes through which venous blood was to be returned to the donor dog. Finally the anterior spinal artery was carefully ligated. The circulation of the recipient's head is maintained only by the bilateral common carotid arteries and vertebral sinuses.
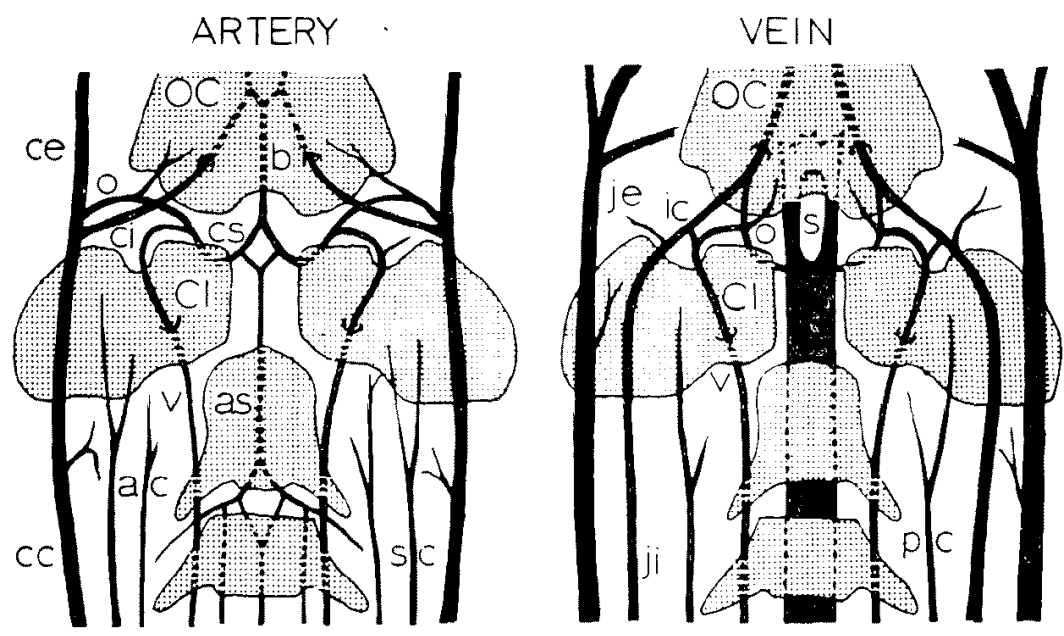

Fig. 1. Principal arteries and veins. Vessels are ligated or clamped at the occipito-atloid level. Muscles and other soft tissues around the neck are transected at the same level, since numerous anastomoses exist or may develop between principal vessels (not shown in diagrams). Bone, OC: occipital, Cl: atlas, whose ventral arch and the dens of the epistropheus are removed. Artery, cc: common carotid, ce: external carotid, ci : internal carotid, o: occipital, ac: ascending cervical, sc: superficial cervical, $v$ : vertebral, $c s$ : cerebrospinal, as: anterior spinal, b: basilar. Vein, je: external jugular, ji : internal jugular, ic: inferior cerebral, pc: profound or deep cervical, v: vertebral, o: occipital, s: vertebral sinus. 
The fitness of the holes, the completeness of the interception of circulation and the appearance of abnormal evidences in the brain should be examined after terminating the experiment.

3. Perfusion Circuit (Fig. 2). An arterial cannula was inserted cranially into one common carotid artery of the recipient dog through which the blood previously held in an arterial blood reservoir was pumped into the head with a pulsatile, constant output pump (modified Dale-Schuster type). The bilateral vertebral sinuses were clamped one after another with special hemostats of curved tip. The venous blood of the recipient's head was led out through the metal cannulae. This venous blood streamed down to the femoral vein of the donor dog via a rotameter and a venous reservoir. Finally another arterial cannula was inserted into the remaining carotid artery. The output of pump is about $1 \mathrm{ml} \times 100$ per min. The circulatory system of head-pump-donor is completely closed against atmosphere.

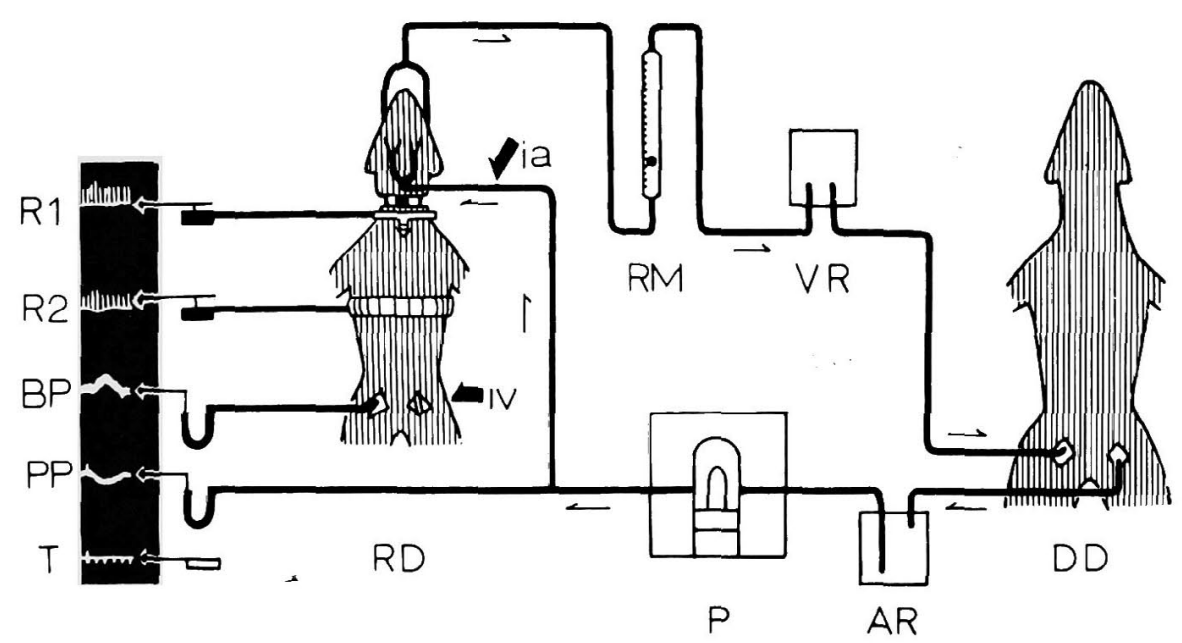

Fig. 2. Schematic representation of perfusion circuit and recording. DD : donor dog, AR: arterial reservoir, P: pump, RD : recipient dog, RM: rotameter, VR: venous reservoir, R1: intratracheal pressure, R2: costo-abdominal movement, BP: systemic blood pressure, PP: perfusion pressure, $\mathrm{T}$ : time base, ia: injection into the head, iv: injection into the trunk.

4. Recording (Fig. 2). Respiration and blood pressure were simultaneously recorded on a smoked paper, i.e., the intratracheal pressure, the costo-abdominal movement, the femoral arterial pressure of the recipient and the perfusion pressure of the head. In addition, EEG and ECG were taken in some preparations.

5. Drug Application. The agent to be tested on the recipient's head was administered into the perfusion circuit leading to the common carotid arteries. On the other hand, to test the effect upon the trunk circulation the drug was injected into the femoral vein of the recipient dog.

In order to assure the completeness of head-trunk circulatory separation, norepinephrine or epinephrine (25-100 $\mu$ g.) was injected into the perfusion circuit. Preparations in which no rise of the recipient's trunk blood pressure was observed after 
norepinephrine into the head were considered to have pharmacologically complete separation of the head and trunk circulations. Likewise, a test dose of norepinephrine or epinephrine was injected into the femoral vein of the recipient (Fig. 3). Preparations in which this test revealed incomplete separation were excluded from consideration in the results.
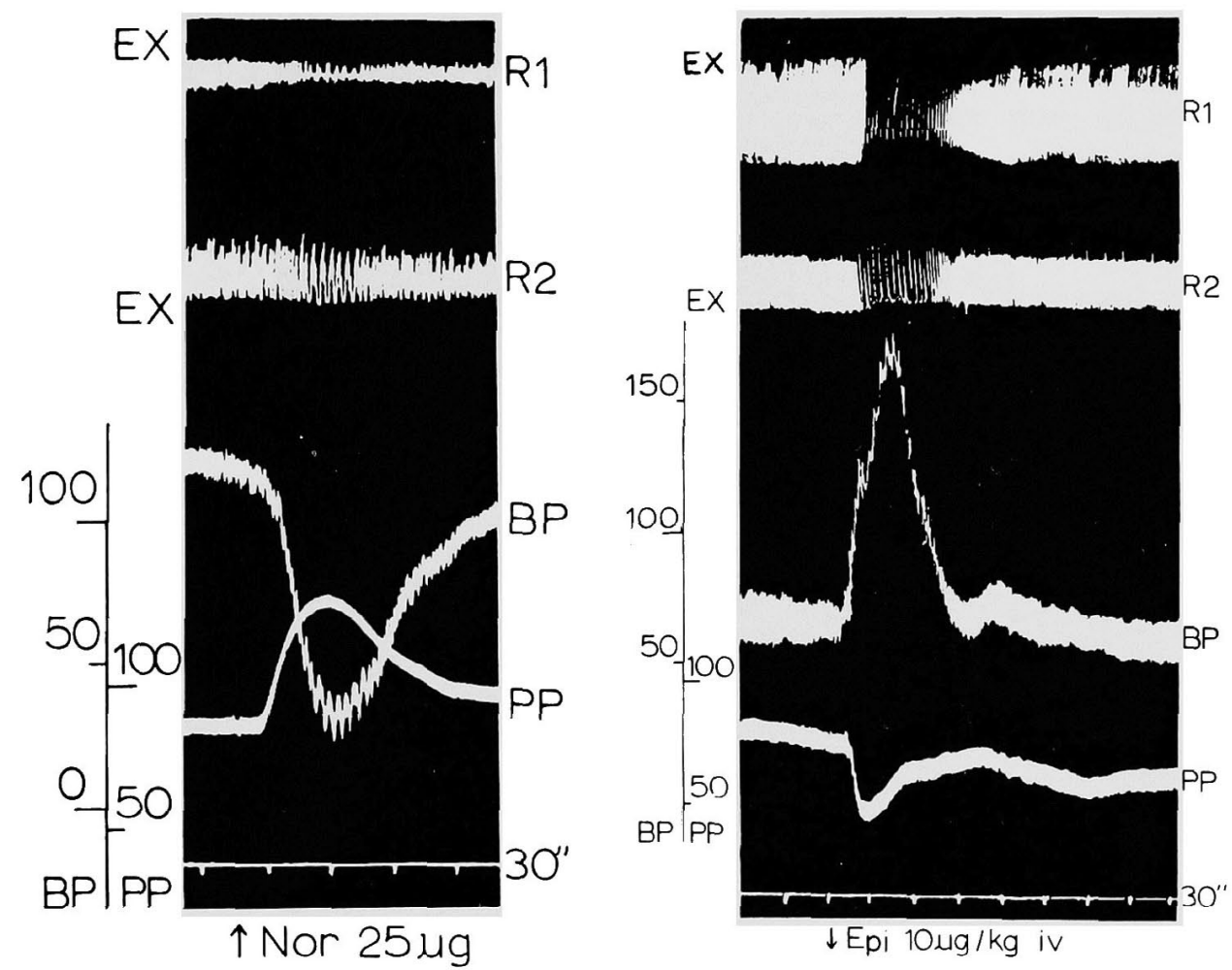

Fig. 3. Test dose of catecholamine. Norepinephrine as well as epinephrine are used in testing circulatory head-trunk separation. In the left panel, norepinephrine is injected into the head producing a rise in PP and reflex hypotension in BP. In the right, epinephrine is given to the trunk producing a rise in $\mathrm{BP}$ and reflex hypotension in PP.

\section{RESUlTS}

1. Phenylalkylamines. The injection of norepinephrine into the head circulation produced a rise in the perfusion pressure. This rise was followed by a fall in the trunk blood pressure (Fig. 3). Even in larger doses of norepinephrine to the head, up to $200 \mu \mathrm{g}$., there was no rise in the trunk blood pressure as seen after systemic administration. Respiration was slightly depressed with or without initial excitation. Sometimes mild excitation of respiration followed the transient depression. Upon the 
denervation of the sinus nerves, reflex fall in the trunk blood pressure due to norepinephrine to the head was strikingly reduced, and the depression of respiration was not so obvious (Fig. 4). The effects of epinephrine were almost similar to those of norepinephrine (Fig. 4); no particular differences between effects of these two agents were observed.

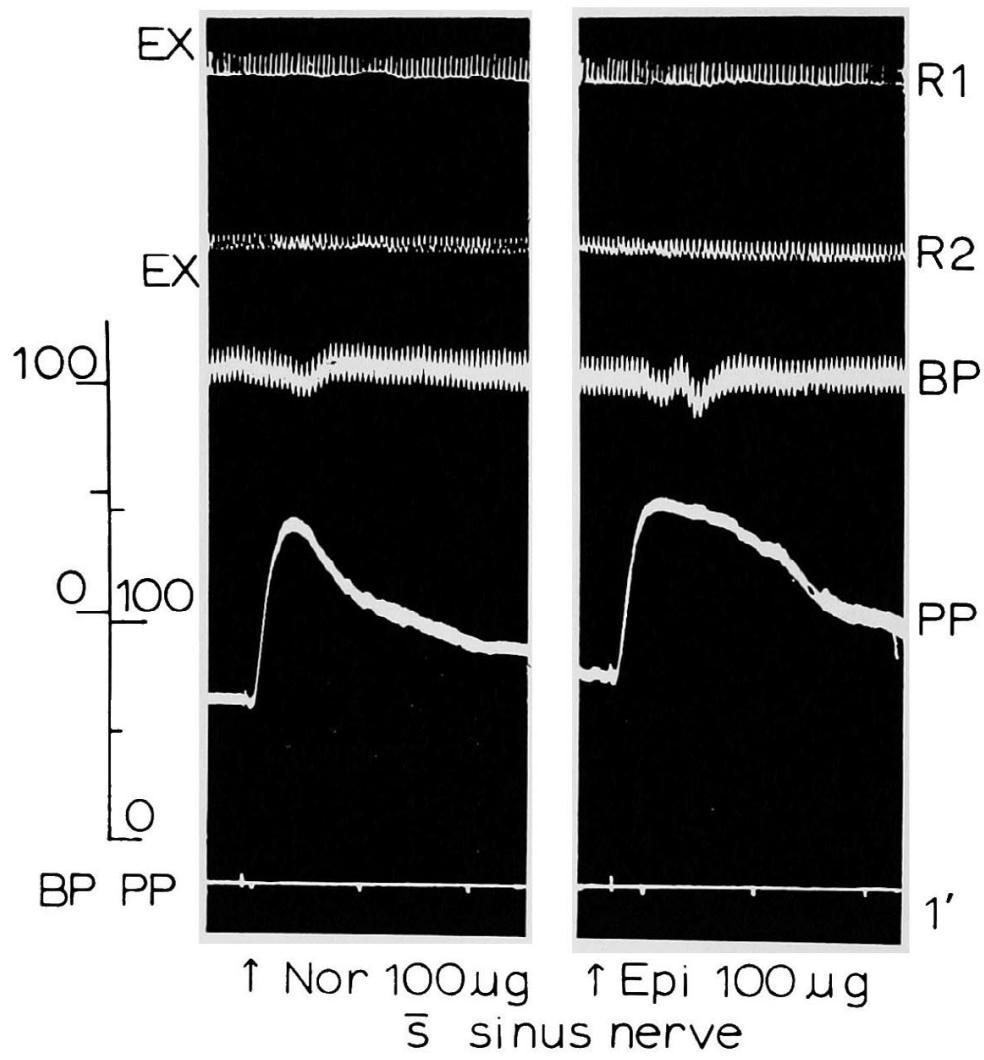

Fig. 4. Catecholamines after sinus denervation. Norepinephrine, in the left, and epinephrine, in the right, produce only minor reflex hypotension in $B P$ after sinus denervation.

The injection of 1 to $20 \mathrm{mg}$. of ephedrine or methamphetamine generally produced a slight fall in perfusion pressure. Correspondingly the systemic blood pressure was slightly elevated. These effects were of longer duration than those after catecholamines. While the respiratory effect of ephedrine was not so marked, methamphetamine produced immediate, moderate respiratory excitation. This excitation did not develop into generalized convulsions as observed following some analepic agents.

2. Acetylcholine and other vasodilator substances. A small dose of 
acetylcholine injected into the head circulation produced a slight rise or only minor fluctuations in the trunk blood pressure. A dose larger than $100 \mu$ g., producing a sharp fall in the head perfusion pressure, caused a rise in the systemic blood pressure and transient hyperpnea. This reflex rise in systemic blood pressure was not abolished by sinus denervation; the respiratory excitation was almost abolished, however (Fig. 5). Pretreatment of the head with atropine considerably reduced the fall in the head blood pressure, and concomitantly reduced the magnitude of the reflex rise in the systemic blood pressure. Quite a large dose of acetylcholine, $1 \mathrm{mg}$., produced a transient, marked hyperpnea which was not abolished by sinus denervation but disappeared after atropinization of the head.

ATP (1-2 mg.), papaverine (1-20 mg.), histamine (0.1-2 mg.), and $\mathrm{NaNO}_{2}$ (1-200 mg.) injected into the head circulation caused similar responses to acetylcholine. Hypertensive reflex response to these drugs, however, was not significantly modified by atropinization. Sudden decrease in arterial blood supply to the head caused a similar responses
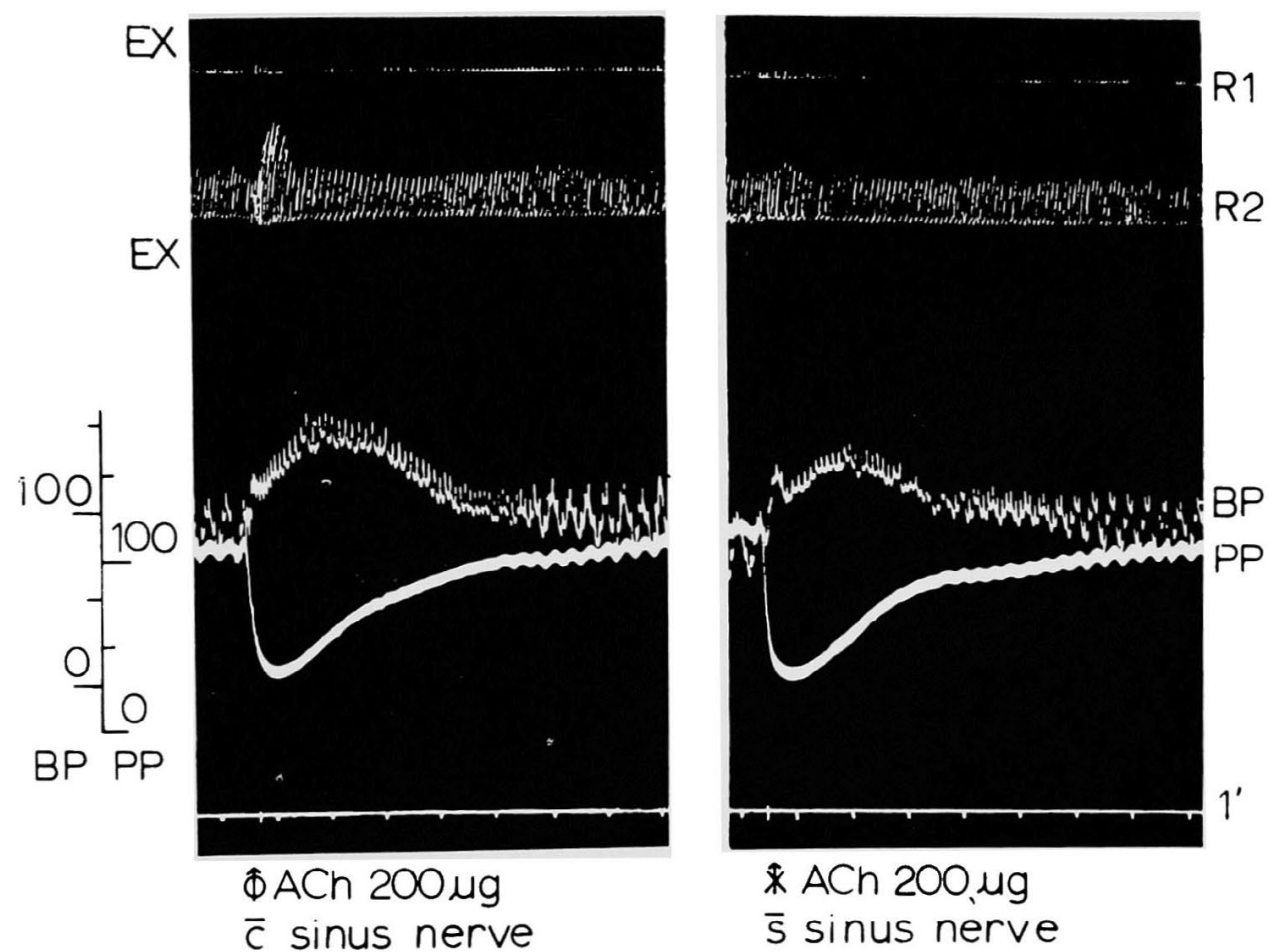

Fig. 5. Acetylcholine effects. In the left, acetylcholine into the head produces transient hyperpnea and reflex rise in BP when the sinus nerve is intact. If it is severed, in the right, acetylcholine produces no hyperpnea but reflex hypertension in BP. 
to these vasodilator substances. In some atypical cases inconsistent or even hypotensive reflex responses were observed after acetylcholine as well as these vasodilators and reduction in blood supply to the head. All these responses were observed regardless of intactness of sinus nerves.

\section{Discussion AND Summary}

The results obtained in this series of experiments do not support any evidence of specific, sensitive chemoreceptors existing in the CNS. The results appear to indicate that the effects of vasoactive drugs may be explained on the basis of alteration in blood supply to the vasomotor centers of the brain or alteration in blood pressure upon some type of baroreceptors, although one must take account of some surgical and chemical influences in interpreting those results obtained in this type of experiments. The decrease or increase of blood supply to the structures directly associated with controlling activity of the cardiovascular system may give rise to elevation or reduction of systemic blood pressure.

It is very interesting to note that the reflex changes in the systemic circulation is much more pronounced when the drug is applied into the head circulation where the internal carotid and occipital arteries are temporarily clamped than where the external carotid artery is clamped. This observation may suggest that the extracranial beds, as a whole, are more sensitive to vasoactive drug than the intracranial beds. When a vasoconstrictor is applied, the extracranial beds may constrict to a higher extent than the intracranial beds, in turn, causing an increase in the blood supply to the intracranial beds. As it were, the vasoconstrictor agent may squeeze the extracranial blood into the intracranial beds. Conversely, a vasodilator substance is likely to withdraw the blood from the intracranial beds, thus causing hypertensive reflex. 